\title{
Intrinsically reinforced silks obtained by incorporation of graphene quantum dots into silkworms
}

\author{
Lin $\mathrm{Ma}^{1,2,3}$, Maxwell Akologo Akurugu ${ }^{1 \dagger}$, Vivian Andoh ${ }^{1 \dagger}$, Haiyan Liu ${ }^{4}$, Jiangchao Song ${ }^{1,2,3}$, \\ Guohua $\mathrm{Wu}^{1,2,3^{*}}$ and Long $\mathrm{Li}^{1,2,3^{*}}$
}

\begin{abstract}
Silkworm silks have been widely used in a variety of fields due to their sensuousness, luster and excellent mechanical properties. Researchers have paid special attention in improving the mechanical properties of silks. In this work, Bombyx mori larval silkworms are injected with graphene quantum dots (GQDs) through a vascular injection to enhance mechanical properties of the silkworm silks. The GQDs can be incorporated into the silkworm silk gland easily due to hemolymph circulation and influence the spinning process of silkworm. The breaking strength, elongation at break and toughness modulus of the silks increase by 2.74, 1.33 and 3.62 times, respectively, by injecting per individual with $0.6 \mu \mathrm{g}$ GQDs. Wide-angle X-ray scattering indicates that the size of $\beta$-sheet nanocrystals in GQDs-silks is smaller than that in control-silks. Infrared spectra suggest that GQDs confine the conformation transition of silk fibroin to $\beta$-sheet from random coil/ $\alpha$-helix, and the change of the size and content of $\beta$ sheet may be the reason for the improvement of the mechanical properties. The toxicity and safety limit of GQDs incorporated into each silkworm is also evaluated, and the results show that the upmost dose of GQDs per silkworm is $30.0 \mu \mathrm{g}$. The successful obtainment of reinforced silks by in vivo uptake of GQDs provides a promising route to produce high-strength silks.
\end{abstract}

Keywords: silkworm silks, graphene quantum dots, decrease of $\beta$-sheets, enhanced mechanical properties

\section{INTRODUCTION}

Silkworm silks (hereafter called silks), spun by silkworms under an aqueous environment, possess exceptional mechanical properties such as great extensibility and high tensile strength, as versatile and tough materials in the world [1-3]. Since discovery, silks have been widely applied in various fields including (but not limited to) textile industry, drug delivery and tissue regeneration [46]. Although silks are tougher than lots of synthetic fibers, their mechanical properties remain to be enhanced compared to spider dragline silks, another natural fiber which are hailed as a "super-fiber" with superior extensibility and strength $[7,8]$. However, raising spiders in a large scale is rather difficult, while silkworms are much milder and can be mass reared $[9,10]$. Much effort has been devoted to improving the performance of silks, which generally falls into two kinds of approaches for functionalized silks, i.e., extrinsical and intrinsical functionalization [11]. Traditional extrinsical functionalization immobilizes certain additives on the surface of silks [12-15], or re-spin silk fibers from regenerated silk fibroin solution with additives [16-18], during which period the structures of silks are easily destroyed due to tedious procedures, harsh condition and the use of toxic reagents. Intrinsical functionalizations including genetic alteration [19-21] of silkworms or in vivo uptake of specific diets by silkworms [22-27] can result in modified silks directly and are much greener. Compared with genetic alteration which needs complicated procedures, the recently developed in vivo uptake method is much easier. Several groups have acquired functionalized silks via adding additives such as dyes [22,23], amino acids [24], bordeaux mixture [25] and nanomaterials [26-31] into the feed of silkworms, among which nanomaterials gain much attention owing to their unique mechanical, antibacterial and photoelectric properties, etc. Cai et al. [26]

\footnotetext{
${ }^{1}$ College of Biotechnology, Jiangsu University of Science and Technology, Zhenjiang 212018, China

${ }^{2}$ The Sericultural Research Institute, Chinese Academy of Agricultural Sciences, Zhenjiang 212018, China

${ }^{3}$ Laboratory of Risk Assessment for Sericultural Products and Edible Insects, Ministry of Agriculture, Zhenjiang 212018, China

${ }^{4}$ Department of tea and food technology, Jiangsu Polytechnic College Agriculture and Forestry, Jurong 212400, China

${ }^{\dagger}$ These authors contributed equally to this work.

* Corresponding authors (emails: georgew511@hotmail.co.uk (Wu G); seri68@hotmail.com (Li L))
} 
demonstrated that silks with improved mechanical properties could be produced by adding $\mathrm{TiO}_{2}$ nanoparticles into the feed of silkworms. Our group [27] found that the mechanical properties of silks could be enhanced significantly by feeding silkworms with mulberry leaves sprayed with copper nanoparticles. Wang and coworkers [28] obtained pristine magnetic silks by feeding silkworms with mulberry leaves sprayed with nano $\mathrm{Fe}_{3} \mathrm{O}_{4}$ powder.

Graphene, which is a material with outstanding mechanical properties, is widely used as reinforcement in high-performance materials [31-35]. Several groups have reported that reinforced silks can be obtained by directly feeding silkworms or spiders with graphene [31,35]. For example, Wang et al. [31] achieved high-strength silks directly by feeding silkworms with graphene nanosheets (with a width of $5 \mu \mathrm{m}$ and a thickness of 6-8 $\mathrm{nm}$ ). Lepore and coworkers [35] revealed that spider silks with enhanced mechanical properties could be obtained by feeding spiders with aqueous dispersions containing graphene flakes (with a diameter of $200-300 \mathrm{~nm}$ ). Graphene quantum dots (GQDs), which are edge-bound nanometer-sized graphene fragments, possess the advantages of both graphene sheets and quantum dots such as strong quantum confinement and edge effects, excellent mechanical properties, large surface area, good biocompatibility and surface grafting [36-39]. Compared with larger graphene nanosheets, zero-dimensional hydrophilic GQDs smaller than $10 \mathrm{~nm}$ are much easier to be incorporated into the crystalline structures of silk fibroins, which may be more suitable for enhancing the mechanical properties of silks.

In this paper, we demonstrated an in vivo uptake (via the method of intravascular injection) of GQDs into domesticated silkworms (bombyx mori), which led to a direct production of intrinsically reinforced silks. Compared with feeding silkworms with GQDs, injection method could ensure that the amount of GQDs each silkworm takes in is clear and equal. The achieved silks exhibit considerably enhanced mechanical properties with superior elongation-at-break and breaking strength, showing the validity of the generation of reinforced silks by the incorporation of GQDs. The as-obtained intrinsically reinforced silks show better mechanical properties than the one produced by feeding with graphene, copper nanoparticle, and silver nanoparticle. The toxicity and the safety limit of GQDs injected for each silkworm were evaluated, and the effect of GQDs on the structures of the obtained silks was also studied by Raman, wide angle X-ray scattering (WAXS) and Fourier transform infrared spectroscopy (FTIR).

\section{EXPERIMENTAL SECTION}

\section{Materials}

The reagents utilized in the whole experiments were of analytical grade. Doubly deionized water was provided by a MilliQ (Elix5+Milli-Q) water purification system (Millipore, Bedford, MA, USA) and was used throughout the experiments. Bombyx mori silkworm eggs were bought from Shandong Guangtong silkworm egg Group Co., Ltd. GQDs with an average diameter of $3.5 \mathrm{~nm}$ were bought from Nanjing XFNANO Materials TECH Co., Ltd.

\section{Characterizations}

The morphology and diameter of degummed silks were characterized by a TESCAN MIRA 3 scanning electron microscope (SEM) (Tescan, Czech Republic). For every sample, 10 single silk fibers were measured. The histological sections of the organs of silkworms were observed by an Olympus BX43 microscope (Olympus, Japan) with a Smart D550 image acquisition system. Raman spectra were collected by a Renishaw inVia laser confocal Raman microspectroscopy (Renishaw inVia, UK), with a $785 \mathrm{~nm}$ excitation laser. WAXS patterns were acquired by a Xeuss 2.0 set-up (Xenocs, France) with the incident X-ray of $\lambda=$ $1.54184 \AA$ A. Version 18 (beta) FIT2D and Peakfit (V4.0) software were used to process the patterns. FTIR was performed on a $670-I R+610-I R$ spectrometer with a diamond attenuated total reflectance accessory (Varian, America), and the calculation of the contents of the protein secondary structures was obtained by deconvolution over the amide I region $\left(1,600-1,700 \mathrm{~cm}^{-1}\right)$. An Instron 3365 material testing instrument (Instron, America) was used for measuring the mechanical properties of degummed silks with an extension rate of $20 \mathrm{~mm} \mathrm{m^{-1 }}$ and a gauge length of $20 \mathrm{~mm}$, with 20 specimens measured for each group. All the silk samples were tested in a constant temperature and humidity room at $20^{\circ} \mathrm{C}$ and $65 \%$ relative humidity $(\mathrm{RH})$.

\section{Silkworm raising and the intake of GQDs}

Bombyx mori silkworms were first hatched from eggs in a climatic chamber and then raised in boxes under the temperature of $26^{\circ} \mathrm{C}$. Fresh mulberry leaves were supplied to the silkworms. The intake of GQDs into silkworms was realized by injecting each silkworm with GQDs using a syringe, or feeding silkworms with mulberry leaves sprayed by GQDs. The injection was conducted on the second day of the fifth instar by injecting each silkworm 
with GQDs at foot (see Scheme S1). Since silkworm has an open circulatory system, it is believed that GQDs will be transferred to the silk gland. As for the feeding method, silkworms were fed with mulberry leaves sprayed by GQDs $\left(0.10 \mathrm{mg} \mathrm{mL}^{-1}\right)$ on the second day of the fifth instar until cocoons were produced. The mass ratio of mulberry leaves to GQDs was about $1 \mathrm{~g}: 0.027 \mathrm{mg}$. According to the intake method and the dose of GQDs taken in each silkworm, the silkworms were divided into 11 groups (each group contains 20 silkworms), designated as GQDs-feeding or GQDs- $x \mu \mathrm{g}$ (here $x$ means the dose of the GQDs injected into each silkworm), i.e., GQDs

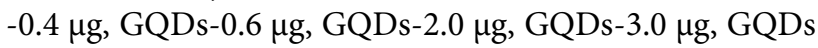
$-6.0 \mu \mathrm{g}$, GQDs-10.0 $\mu \mathrm{g}$, GQDs-30.0 $\mu \mathrm{g}$, GQDs-50.0 $\mu \mathrm{g}$ and GQDs-100.0 $\mu \mathrm{g}$. The control group was designed by injecting per silkworm with $10 \mu \mathrm{L}$ distilled water. The details for the intake of GQDs into silkworms are also supplied in Supplementary information (SI).

\section{Silk reeling}

The obtained cocoons were reeled to remove the sericin coated on the silks. Cocoons were dried for $4 \mathrm{~h}$ at $80^{\circ} \mathrm{C}$ in a vacuum drying oven. The dried cocoons were softened in boiling water for a few minutes, which were then transferred into hot water $\left(70^{\circ} \mathrm{C}\right)$. After that, the treated cocoons were reeled using a XJ401 automatic cocoon reeling apparatus (Hangzhou Feiyu technological engineering Co., Ltd., China). It should be noted that seven cocoons were reeled together at a time, and the finally obtained degummed silk fiber comprised seven single silks.

\section{RESULTS AND DISCUSSION}

\section{Toxicity and safety limit of GQDs to silkworm}

In this assay, two hundred silkworms injected with different doses of GQDs were divided into ten groups evenly (called control, GQDs-0.4 $\mu \mathrm{g}$, GQDs-0.6 $\mu \mathrm{g}$, GQDs$2.0 \mu \mathrm{g}$, GQDs-3.0 $\mu \mathrm{g}$, GQDs-6.0 $\mu \mathrm{g}$, GQDs-10.0 $\mu \mathrm{g}$, GQDs-30.0 $\mu \mathrm{g}$, GQDs-50.0 $\mu \mathrm{g}$ and GQDs-100.0 $\mu \mathrm{g}$, respectively). In addition, twenty silkworms were fed with mulberry leaves sprayed by GQDs as a comparison (named GQDs-feeding). In order to reveal the toxicity and safety limit of GQDs to silkworm, the mortality rate (MR) for each group was observed (Table S2) after the intake of GQDs with $24 \mathrm{~h}$ intervals $(24,48,72,96$ and $120 \mathrm{~h}$ ). It exhibits that the MR for every group is almost 0 when the GQDs dose (per silkworm) is limited to 0-30.0 $\mu \mathrm{g}$, when the GQDs dose (per silkworm) increases beyond $50.0 \mu \mathrm{g}$, the MR of both GQDs-group and their corresponding control-group increases significantly, indicating that the safety limit of GQDs to silkworm is in the range 30.0-50.0 $\mu \mathrm{g}$ per silkworm. Histophysiological evaluation was carried out to further study the toxicity of GQDs at the dose of $30.0 \mu \mathrm{g}$ (per silkworm), the results in Fig. S2 illustrate that GQDs has no negative effects on the pathological structures of silkworm organs (midgut, liposome and posterior silk gland), indicating that GQDs at the dose of $30.0 \mu \mathrm{g}$ (per silkworm) is of no or very low toxicity. Since the doses of 50.0 and $100.0 \mu \mathrm{g}$ (per silkworm) are dangerous to silkworms, neither GQDs$50.0 \mu \mathrm{g}$ nor GQDs-100.0 $\mu \mathrm{g}$ group is further investigated in the following text.

The effect of GQDs on the growth and silks of silkworms was also studied as shown in Fig. 1. It reveals that the average weight (AW) of every GQDs-injection-group is lower than the one of GQDs-feeding group, while the average length (AL) shows no obvious difference (Fig. 1a and $\mathrm{b}$, Table S3); the decrease of AW may be caused by the injection injury. It is found that the AW of GQDs$4.0 \mu \mathrm{g}$ group is lower than those of other GQDs-injectiongroups, it is mainly due to that the silkworms initially chosen for this group were lighter, because it is observed that its AW before injection is also lower. The appearance of the silkworms was also observed after intake of GQDs for $72 \mathrm{~h}$ (Fig. 1c). Furthermore, the morphology and diameter of the silks of each group were studied by SEM. It should be noted that the silks hereafter discussed are all degummed silks, which means that the sericin coated on the silks are removed. From Fig. 1d, it can be seen that the silks exhibit similar morphology, indicating that the incorporation of GQDs does not affect the silk morphology obviously. However, the average diameter (AD) of silks from the control-group is a little larger than the one of GQDs-groups (Table S3). Since GQDs were taken in by silkworms and could spread into silk gland, the interactions between GQDs and silk fibroin may influence the spinning process of the silkworms, leading to a slight decrease of the $\mathrm{AD}$ of silks. The confirmation of the GQDs in silkworm and silk gland was provided in SI.

\section{Characterization of the structural and conformational changes of silks}

The effect of GQDs on the structural and conformational changes of silks was studied with Raman spectroscopy, WAXS and FTIR.

\section{Raman spectra}

Raman spectroscopy was used to study the conformational changes of the silks upon the intake of GQDs in the 

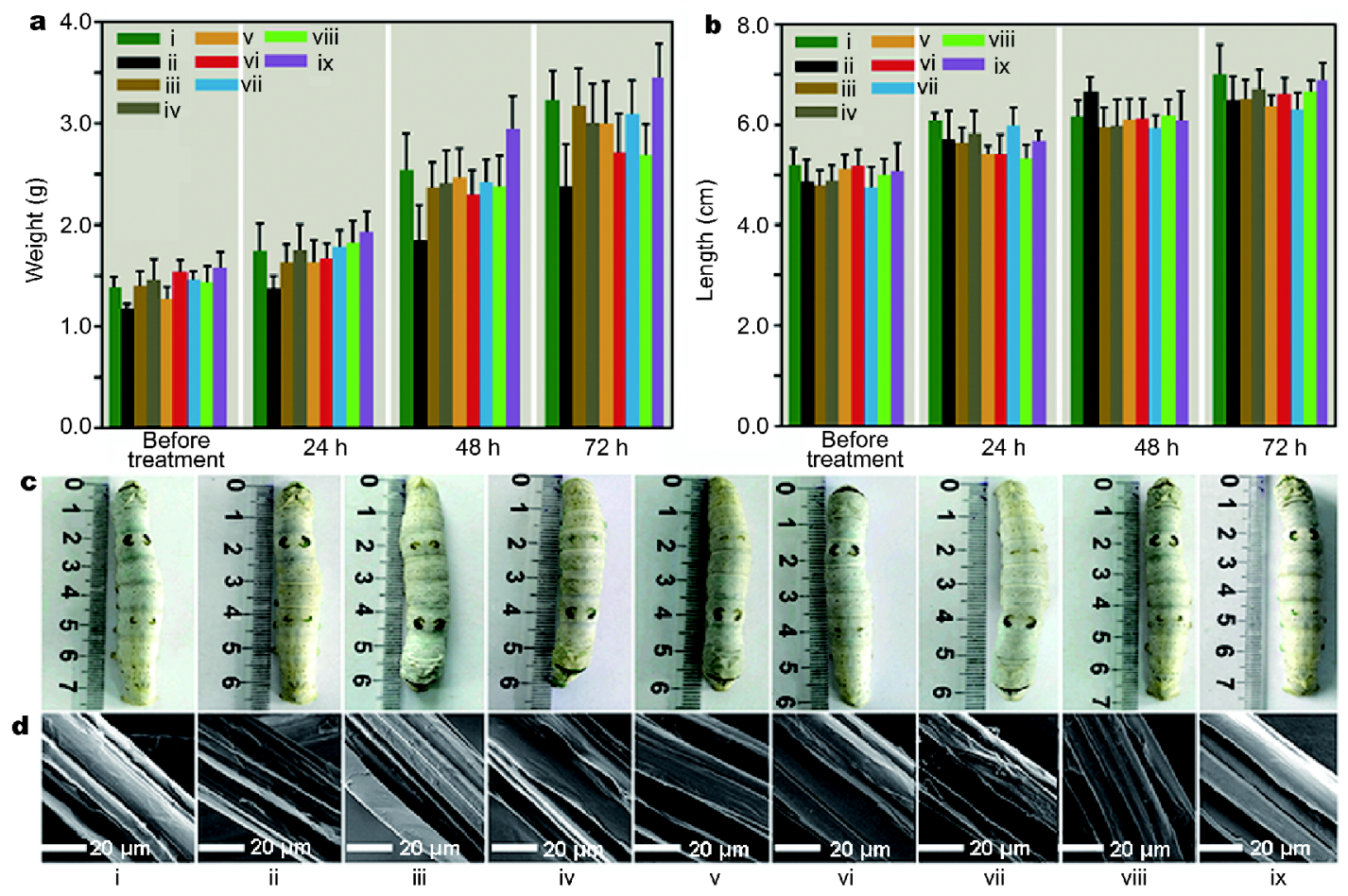

Figure 1 The effect of GQDs on the growth and silks of silkworms. (a, b) The effect of GQDs on the average weight (a) and length (b) of silkworms after injection or feeding with GQDs for different time. (c) The effect of GQDs on the appearance of silkworms after injection or feeding with GQDs for $72 \mathrm{~h}$. (d) The effect of GQDs on degummed silks. From i to ix, the silkworms or silk samples are control (i), GQDs-0.4 $\mu \mathrm{g}$ (ii), GQDs-0.6 $\mu \mathrm{g}$ (iii), GQDs-2.0 $\mu \mathrm{g}$ (iv), GQDs-3.0 $\mu \mathrm{g}$ (v), GQDs-6.0 $\mu \mathrm{g}$ (vi), GQDs-10.0 $\mu \mathrm{g}$ (vii), GQDs-30.0 $\mu \mathrm{g}$ (viii) and GQDs-feeding groups (ix), respectively.

region of $800-4,000 \mathrm{~cm}^{-1}$, as shown in Fig. 2. It is observed that the most prominent Raman-active bands for silks from either control- or GQDs-groups are located at the same positions including $1,087 \mathrm{~cm}^{-1}$ (random coil conformation), $1,232 \mathrm{~cm}^{-1}$ (predominantly $\beta$-sheet conformation) and $1,669 \mathrm{~cm}^{-1}$ ( $\beta$-sheet $/ \beta$-turn conformation) $[40,41]$, indicating that the conformation of silks are not changed with the intake of GQDs. In addition, it is found that the characteristic Raman bands of GQDs-silks have stronger intensities than that of control-silks. Raman features corresponding to GQDs are not observed in the Raman spectra, because the amount of GQDs in silk is too low.

\section{WAXS spectra}

The influence of GQDs on the crystalline structures of silks was studied by WAXS, the corresponding $2 \mathrm{D}$ - and 1D-WAXS patterns are shown in Figs 3-5, respectively. It can be observed that both the 2D- and 1D-WAXS patterns do not show any obvious difference among all the silk samples, proving that the basic structures of silks are not destroyed by the intake of GQDs, which is consistent with the results from Raman spectra. The mean crystallite

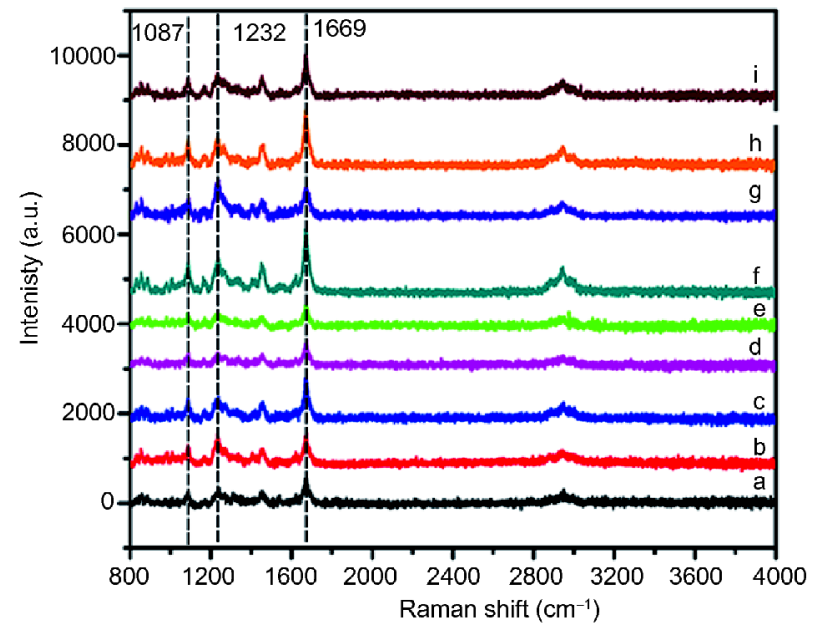

Figure 2 Raman spectra of different silk samples. From a to i, the silks are control (a), GQDs-0.4 $\mathrm{g}$ (b), GQDs-0.6 $\mu \mathrm{g}$ (c), GQDs-2.0 $\mu \mathrm{g}$ (d), GQDs-3.0 $\mu \mathrm{g}$ (e), GQDs-6.0 $\mu \mathrm{g}$ (f), GQDs-10.0 $\mu \mathrm{g}$ (g), GQDs-30.0 $\mu \mathrm{g}(\mathrm{h})$ and GQDs-feeding groups (i), respectively.

size of $\beta$-sheets in $a$ direction (interchain), $b$ direction (intersheet) and $c$ direction (along fiber axis), which is directly related to the mechanical properties of silks [42], 


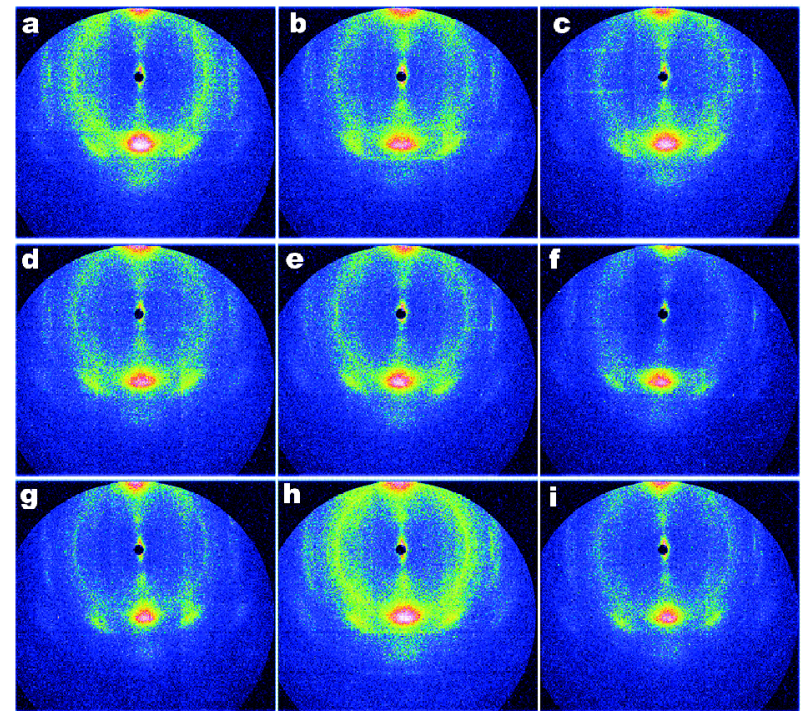

Figure 3 2D-WAXS patterns of different silk samples. From a to i, the silks are control (a), GQDs-0.4 $\mu \mathrm{g}$ (b), GQDs-0.6 $\mu \mathrm{g}$ (c), GQDs-2.0 $\mu \mathrm{g}$ (d), GQDs-3.0 $\mu \mathrm{g}$ (e), GQDs-6.0 $\mu \mathrm{g}$ (f), GQDs-10.0 $\mu \mathrm{g}$ (g), GQDs $-30.0 \mu \mathrm{g}(\mathrm{h})$ and GQDs-feeding groups (i), respectively. was obtained by analyzing the main equatorial (200), (120) and meridian (002) reflections [43-46], respectively, through Scherrer formula $[47,48]$. The results shown in Table S4 demonstrate that the $\beta$-sheet nanocrystal size of GQDs-silks is smaller than that of controlsilk in $a, b$ and $c$ direction. The variation of the $\beta$-sheet nanocrystal size in all the three directions among the GQDs-silks has no conspicuous regularity.

\section{FTIR spectra}

FTIR, which is one of the most effective techniques for investigating the secondary structures of silks $[49,50]$, was also used to study the superior inter-structures of the silks. As shown in Fig. 6a, the control- and GQDs-silks have the identical peak positions in their respective FTIR spectra, confirming that GQDs do not change the secondary structures of silks. The assignment of the three significant peaks located at 1,618 (amide I), 1,514 (amide II) and $1,230 \mathrm{~cm}^{-1}$ (amide III) in the spectra is shown in Table S5. In this paper, the amide I spectral region $\left(1,600-1,700 \mathrm{~cm}^{-1}\right)$ was employed to analyze the silk
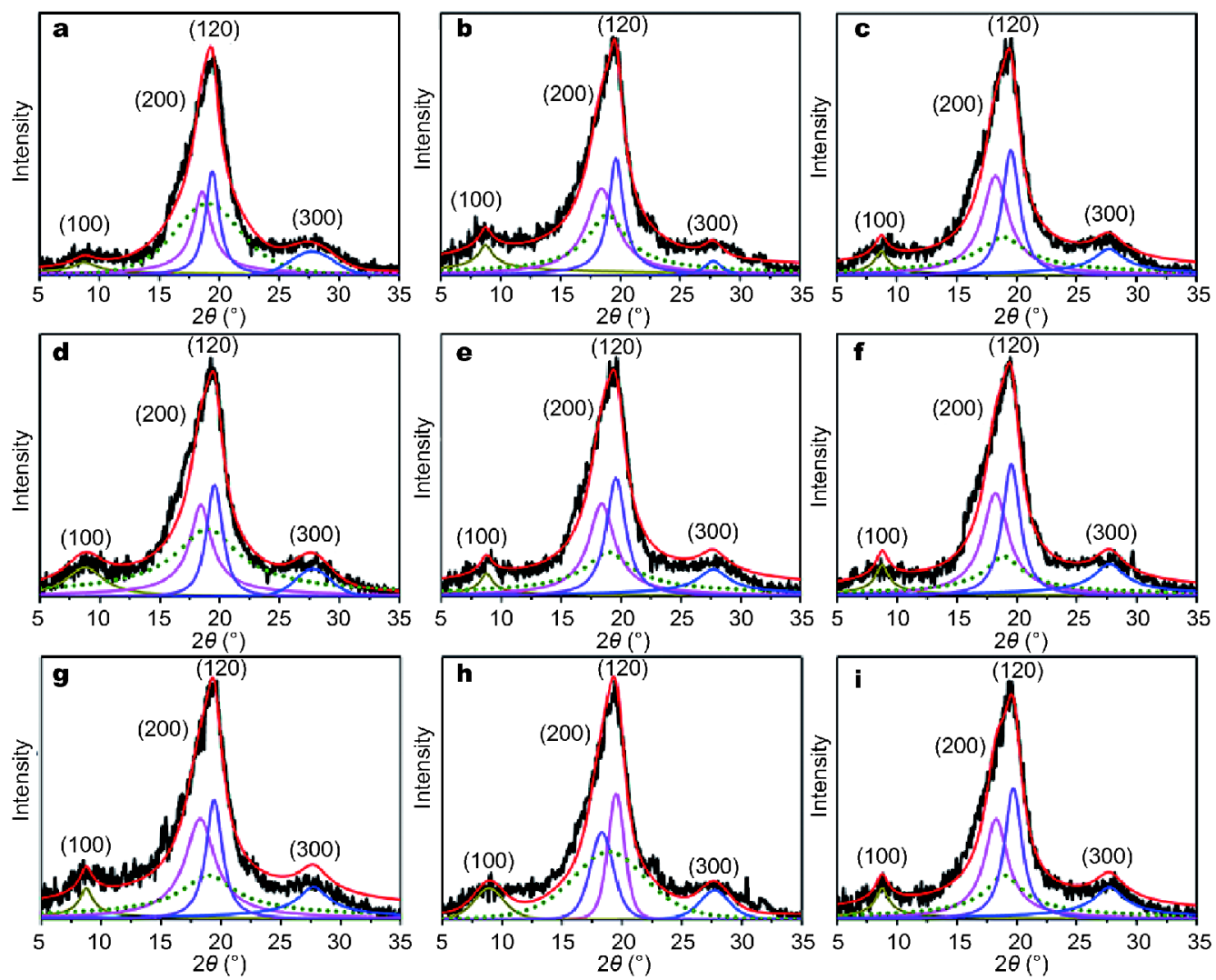

Figure 4 1D-WAXS profiles along the equatorial direction from the 2D-WAXS patterns shown in Fig. 3, with deconvolutions of the underlying

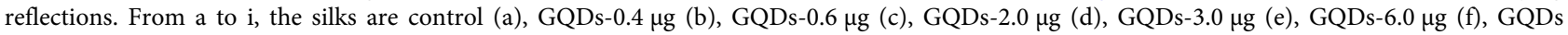
$-10.0 \mu \mathrm{g}(\mathrm{g})$, GQDs-30.0 $\mu \mathrm{g}(\mathrm{h})$ and GQDs-feeding groups (i), respectively. 

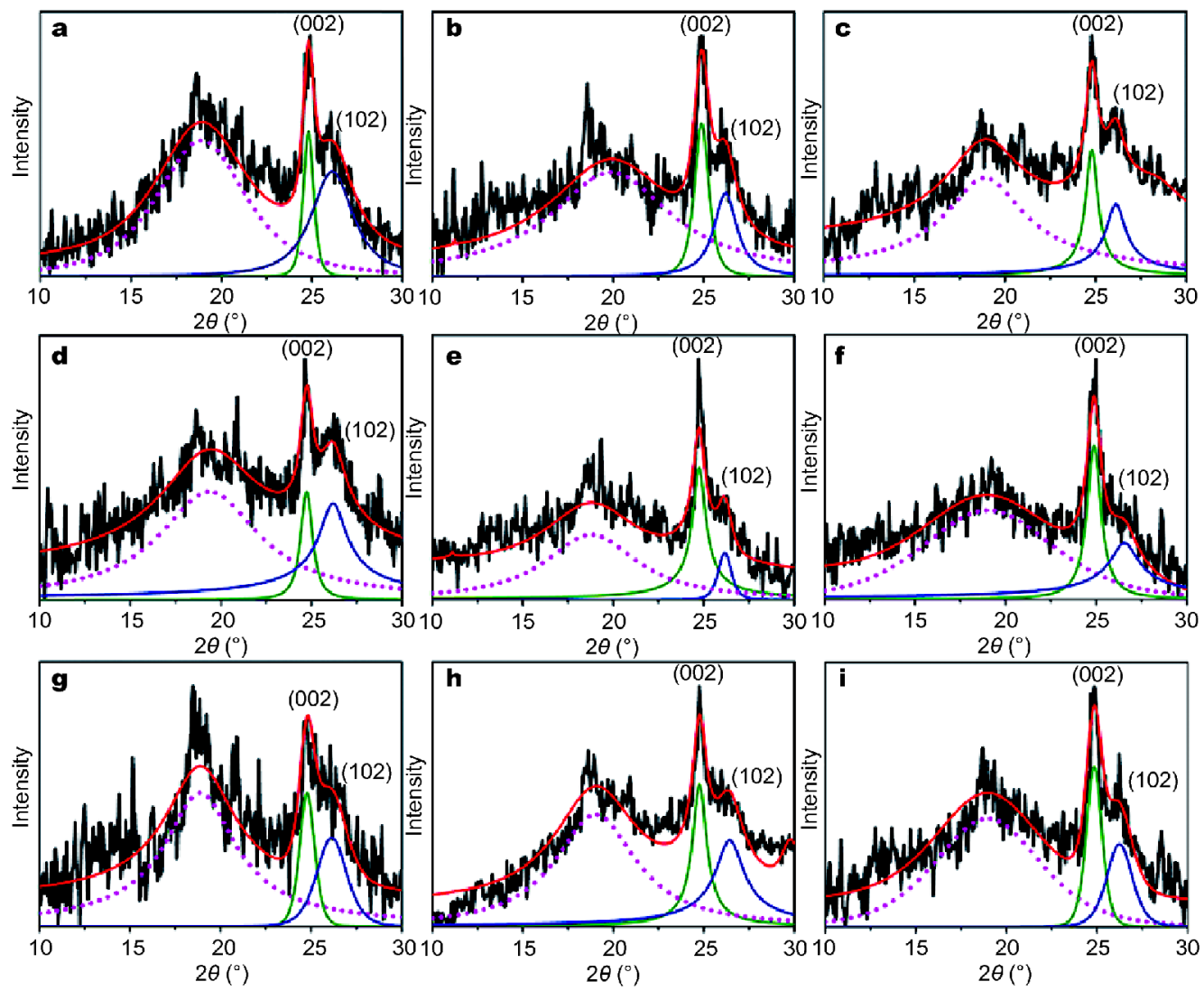

Figure 5 1D-WAXS profiles along the meridian direction from the 2D-WAXS patterns shown in Fig. 3, with deconvolutions of the underlying

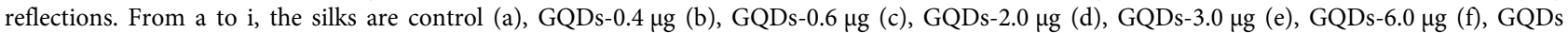
$-10.0 \mu \mathrm{g}(\mathrm{g})$, GQDs-30.0 $\mu \mathrm{g}(\mathrm{h})$ and GQDs-feeding groups (i), respectively.
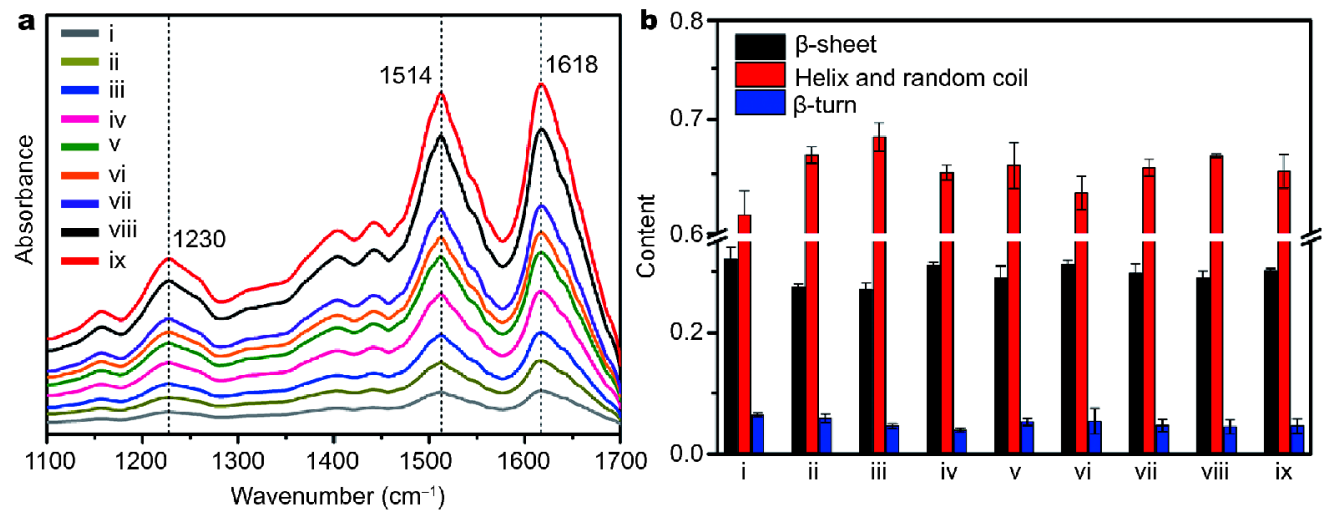

Figure 6 Influence of GQDs on the secondary structures of silks. (a) FTIR spectra of different silk samples and (b) the content of secondary structures of different silk samples according to the amide I band in Fig. 6a. From i to ix, the samples are control (i), GQDs-0.4 $\mu \mathrm{g}$ (ii), GQDs-0.6 $\mu \mathrm{g}$ (iii), GQDs $-2.0 \mu \mathrm{g}$ (iv), GQDs-3.0 $\mu \mathrm{g}$ (v), GQDs-6.0 $\mu \mathrm{g}$ (vi), GQDs-10.0 $\mu \mathrm{g}$ (vii), GQDs-30.0 $\mu \mathrm{g}$ (viii) and GQDs-feeding groups (ix), respectively.

secondary structures. The peak located at the range of 1,615 to $1,640 \mathrm{~cm}^{-1}$ is considered to be the $\beta$-sheet structures, the peak at the range of 1,640 to $1,660 \mathrm{~cm}^{-1}$ is attributed to be the helical or random coil conformation or both and the peak centered at $1,690 \mathrm{~cm}^{-1}$ is classified as $\beta$-turn conformations [49-51]. The amide I spectral region was de-convoluted to determine the contents of the secondary structures, and the details in different silk 


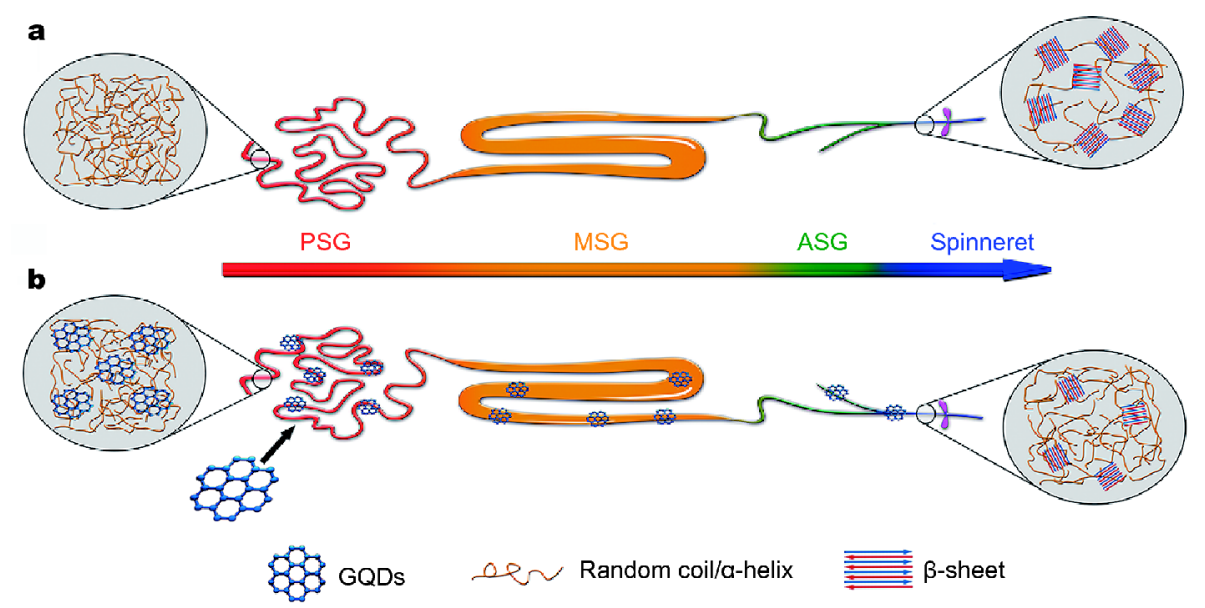

Scheme 1 Schematic diagram shows (a) the transformation of $\alpha$-helix/random coils to $\beta$-sheets in silk gland and (b) the influence of GQDs on the transformation of $\alpha$-helix/random coils to $\beta$-sheets in silk gland. PSG refers to posterior silk gland, MSG refers to middle silk gland, and ASG refers to anterior silk gland.

samples can be found in Fig. S4. The contents of the secondary structures are shown in Fig. $6 \mathrm{~b}$ and Table S6. The GQDs-silks have more helix and random coil and fewer $\beta$-sheet and $\beta$-turn than control-silks. Generally, with the increase of the amount of GQDs, the helix and random coil in GQDs-silks show a tendency of rising up first and declining later, while the $\beta$-sheet and $\beta$-turn display an opposite tendency. The detailed explanation for the variation trend of the content of secondary structure still remains a challenge to us. We hope that the mechanism can be revealed by further study.

It is believed that GQDs can be spread and accumulated into silk gland by passing across epithelial tissue [11]. In silk gland, the hydrophilic GQDs with high specific surface area and rich surface active sites [37] could interact with the fibroin matrix via hydrophobic, electrostatic, van der Waals, hydrogen bonding or $\pi-\pi$ stacking interactions [52-55], which may influence the migration process of fibroin in posterior gland to spinneret and hinder the $\alpha$ helix and random coils from transforming into $\beta$-sheets, leading to a decrease of the $\beta$-sheets. Scheme 1 illustrates the influence of GQDs on the transformation of $\alpha$-helix and random coils to $\beta$-sheets.

\section{Mechanical properties}

The mechanical properties (including breaking strength, elongation at break and toughness modulus) of silks were closely related to their secondary structures $[42,56]$. From Fig. 7 and Table 1, it is observed that GQDs-silks have significantly improved mechanical properties compared with control-silks. GQDs-0.6 $\mu$ g-silks exhibit the highest breaking strength of $675.50 \mathrm{MPa}$ (elongation at break is

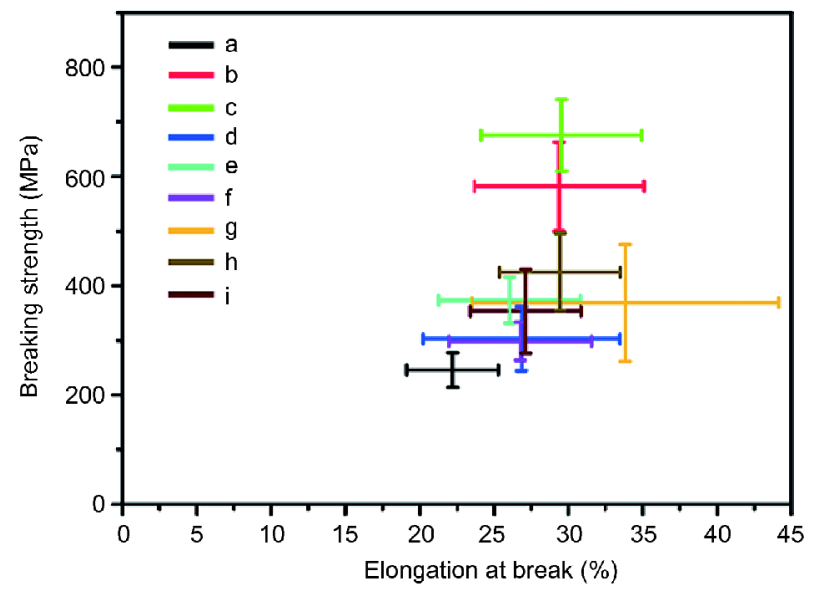

Figure 7 The elongation at break-breaking strength behavior of different silk samples. The error bars exhibit the standard deviation of elongation at break (abscissa) and breaking strength (ordinate). From a to $i$, the samples are control (a), GQDs-0.4 $\mu \mathrm{g}$ (b), GQDs-0.6 $\mu \mathrm{g}$ (c), GQDs-2.0 $\mu \mathrm{g}$ (d), GQDs-3.0 $\mu \mathrm{g}$ (e), GQDs-6.0 $\mu \mathrm{g}$ (f), GQDs-10.0 $\mu \mathrm{g}$ (g), GQDs-30.0 $\mu \mathrm{g}(\mathrm{h})$ and GQDs-feeding groups (i), respectively.

29.53\%) and GQDs-10.0 $\mu \mathrm{g}$-silks possess the best elongation at break of $34.56 \%$ (breaking strength is $369.20 \mathrm{MPa}$ ), considerably exceeding the ones of control silks (246.28 MPa, 22.19\%). The toughness modulus, defined as the area under the stress-strain curve (Fig. S5), is 143.51 MPa for GQDs-0.6 $\mu$ g-silks, showing a 3.62-fold increase compared with that of control-silk. It is found that with the increase of the GQDs dosages, the average mechanical properties values of GQDs-silks rise first and descend later (Fig. S6), indicating that excessive GQDs result in deteriorated mechanical properties of silks. 
Table 1 Mechanical properties of different silk samples

\begin{tabular}{cccc}
\hline Sample & $\begin{array}{c}\text { Breaking } \\
\text { strength (MPa) }\end{array}$ & $\begin{array}{c}\text { Elongation at } \\
\text { break (\%) }\end{array}$ & $\begin{array}{c}\text { Toughness } \\
\text { modulus (MPa) }\end{array}$ \\
\hline Control & 246.28 & 22.19 & 39.66 \\
GQDs-0.4 $\mu \mathrm{g}$ & 582.03 & 29.37 & 123.20 \\
GQDs-0.6 $\mu \mathrm{g}$ & 675.50 & 29.53 & 143.51 \\
GQDs-2.0 $\mu \mathrm{g}$ & 303.16 & 26.85 & 59.51 \\
GQDs-3.0 $\mu \mathrm{g}$ & 373.83 & 26.04 & 68.54 \\
GQDs-6.0 $\mu \mathrm{g}$ & 298.35 & 26.78 & 56.59 \\
GQDs-10.0 $\mu \mathrm{g}$ & 369.20 & 34.56 & 96.09 \\
GQDs-30.0 $\mu \mathrm{g}$ & 424.98 & 29.43 & 90.05 \\
GQDs-feeding & 353.48 & 27.12 & 69.35 \\
\hline
\end{tabular}

The enhancement of breaking strength may be due to the decrease of $\beta$-sheet nanocrystal size. It was reported that the hydrogen bonds in $\beta$-sheet crystal were loaded in shear in small nanocrystals while in tension in large crystals; since hydrogen bonds were greatly weaker in non-uniform tension than in uniform shear, they consequently showed a significantly varied ability to withstand deformation, i.e., smaller $\beta$-sheet nanocrystals can dissipate obviously more energy during failure, leading to a higher fracture toughness $[42,56]$. However, it is noted that the changing tendency of breaking strength is not consistent with the one of $\beta$-sheet nanocrystal size, with the variation of the amount of GQDs. This may be attributed to the fact that the breaking strength of silks can be influenced by multiple factors such as the nanofibrils or nanodomains, $\beta$-sheet content, the size and distribution of the crystallites and the orientational organization at the molecular level [57-59]. The change of the $\beta$-sheet nanocrystal size will lead to the variation of the breaking strength of silks but is not the only factor. The increase of elongation at break and toughness modules is believed to be caused by the higher content of helix and random coil in GQDs-silks, because helix and random coil conformation possess more easily movable chains than $\beta$ sheet [31]. However, the excessive GQDs may aggregate and act as defects, leading to a low breaking strength or elongation at break [31].

It should be noted that the control-silks in this study show a lower breaking strength $(246.28 \mathrm{MPa})$ compared with the typical commercial silks $(300-700 \mathrm{MPa})[7,27]$, the difference should be attributed to the variety of silkworms [27]. It was reported that the mechanical properties of silks were closely related to the rearing environment, spinning speed, degumming process, test- ing parameters, etc. [7,31], Therefore, our silkworms were all reared in the same environment and the silks were all reeled under the same condition.

\section{CONCLUSIONS}

This study shows that intrinsically reinforced silks could be obtained via injecting silkworms with GQDs. Compared with control silks, the breaking strength $(675.5 \mathrm{MPa})$ and elongation-at-break (29.53\%) of the GQDs-0.6 $\mu \mathrm{g}$-silks increase to 2.74 and 1.33 times on average, respectively. The toxicity and safety limit of GQDs are evaluated, and the result reveals that GQDs exhibits no toxicity when the dose of GQDs is less than $30.0 \mu \mathrm{g} /$ silkworm. The WAXS, Raman and FTIR spectra suggest that compared with control silks, the secondary structures of GQDs-silks are well preserved while with fewer $\beta$-sheet and more $\alpha$-helix/random coil structures, which may enhance the mechanical properties of silks. The intake of GQDs will influence the fibrillation of liquid silk fibroin in silk gland, hindering the transformation of hydrogen bonding from intra-molecular to inter-molecular in silk fibroin. In addition, we also compare the growth status (including weight and length) with silks' morphology, diameter and mechanical properties of the silkworms treated by injecting and feeding methods, which demonstrates that injecting method does not have negative effects on the silks. This work introduces a new material for the improvement of mechanical properties of silks. However, there are still some problems which remain challenges, for example how GQDs influences the structures of silks in biological process. In addition, compared with feeding silkworms directly with GQDs, the injecting method is obviously not suitable to produce silks on a large scale. Further researches concerning these problems need to be done.

Received 10 March 2018; accepted 4 June 2018; published online 11 July 2018

1 Guo C, Zhang J, Wang X, et al. Comparative study of straindependent structural changes of silkworm silks: insight into the structural origin of strain-stiffening. Small, 2017, 13: 1702266

2 Wang X, Zhao P, Li Y, et al. Modifying the mechanical properties of silk fiber by genetically disrupting the ionic environment for silk formation. Biomacromolecules, 2015, 16: 3119-3125

3 Koh LD, Cheng Y, Teng CP, et al. Structures, mechanical properties and applications of silk fibroin materials. Prog Polymer Sci, 2015, 46: 86-110

4 Fang G, Sapru S, Behera S, et al. Exploration of the tight structural-mechanical relationship in mulberry and non-mulberry silkworm silks. J Mater Chem B, 2016, 4: 4337-4347

5 Li G, Li Y, Chen G, et al. Silk-based biomaterials in biomedical 
textiles and fiber-based implants. Adv Healthcare Mater, 2015, 4: 1134-1151

6 Iizuka T, Sezutsu H, Tatematsu K, et al. Colored fluorescent silk made by transgenic silkworms. Adv Funct Mater, 2013, 23: 52325239

7 Blamires SJ, Blackledge TA, Tso IM. Physicochemical property variation in spider silk: ecology, evolution, and synthetic production. Annu Rev Entomol, 2017, 62: 443-460

8 Florczak A, Jastrzebska K, Mackiewicz A, et al. Blending two bioengineered spider silks to develop cancer targeting spheres. J Mater Chem B, 2017, 5: 3000-3011

9 Teulé F, Miao YG, Sohn BH, et al. Silkworms transformed with chimeric silkworm/spider silk genes spin composite silk fibers with improved mechanical properties. Proc Natl Acad Sci USA, 2012, 109: 923-928

10 Andersson M, Jia Q, Abella A, et al. Biomimetic spinning of artificial spider silk from a chimeric minispidroin. Nat Chem Biol, 2017, 13: 262-264

11 Tansil NC, Koh LD, Han MY. Functional silk: colored and luminescent. Adv Mater, 2012, 24: 1388-1397

$12 \mathrm{Lu} \mathrm{Z}$, Mao C, Meng M, et al. Fabrication of $\mathrm{CeO}_{2}$ nanoparticlemodified silk for UV protection and antibacterial applications. J Colloid Interface Sci, 2014, 435: 8-14

13 Chang S, Kang B, Dai Y, et al. A novel route to synthesize CdS quantum dots on the surface of silk fibers via $\gamma$-radiation. Mater Lett, 2008, 62: 3447-3449

14 Wang X, Gao W, Xu S, et al. Luminescent fibers: In situ synthesis of silver nanoclusters on silk via ultraviolet light-induced reduction and their antibacterial activity. Chem Eng J, 2012, 210: 585-589

15 Zhang P, Lan J, Wang Y, et al. Luminescent golden silk and fabric through in situ chemically coating pristine-silk with gold nanoclusters. Biomaterials, 2015, 36: 26-32

16 Zhang F, Lu Q, Yue X, et al. Regeneration of high-quality silk fibroin fiber by wet spinning from $\mathrm{CaCl}_{2}$-formic acid solvent. Acta Biomater, 2015, 12: 139-145

$17 \mathrm{Hu} \mathrm{X}, \mathrm{Li}$ J, Bai Y. Fabrication of high strength graphene/regenerated silk fibroin composite fibers by wet spinning. Mater Lett, 2017, 194: 224-226

18 Zhang $\mathrm{C}$, Zhang $\mathrm{Y}$, Shao $\mathrm{H}$, et al. Hybrid silk fibers dry-spun from regenerated silk fibroin/graphene oxide aqueous solutions. ACS Appl Mater Interfaces, 2016, 8: 3349-3358

19 Tamura T, Thibert C, Royer C, et al. Germline transformation of the silkworm Bombyx mori L. using a piggyBac transposon-derived vector. Nat Biotechnol, 2000, 18: 81-84

20 Wang F, Xu H, Wang Y, et al. Advanced silk material spun by a transgenic silkworm promotes cell proliferation for biomedical application. Acta Biomater, 2014, 10: 4947-4955

21 Wen H, Lan X, Zhang Y, et al. Transgenic silkworms (Bombyx mori) produce recombinant spider dragline silk in cocoons. Mol Biol Rep, 2010, 37: 1815-1821

22 Nisal A, Trivedy K, Mohammad H, et al. Uptake of azo dyes into silk glands for production of colored silk cocoons using a green feeding approach. ACS Sustain Chem Eng, 2014, 2: 312-317

23 Tansil NC, Li Y, Teng CP, et al. Intrinsically colored and luminescent silk. Adv Mater, 2011, 23: 1463-1466

24 Teramoto H, Kojima K. Production of Bombyx mori silk fibroin incorporated with unnatural amino acids. Biomacromolecules, 2014, 15: 2682-2690

25 Fernandes J, Nicodemo D, Oliveira JE, et al. Enhanced silk performance by enriching the silkworm diet with bordeaux mixture. J
Mater Sci, 2017, 52: 2684-2693

26 Cai L, Shao H, Hu X, et al. Reinforced and ultraviolet resistant silks from silkworms fed with titanium dioxide nanoparticles. ACS Sustain Chem Eng, 2015, 3: 2551-2557

27 Wu GH, Song P, Zhang DY, et al. Robust composite silk fibers pulled out of silkworms directly fed with nanoparticles. Int J Biol Macromolecules, 2017, 104: 533-538

28 Wang JT, Li LL, Feng L, et al. Directly obtaining pristine magnetic silk fibers from silkworm. Int J Biol Macromolecules, 2014, 63: 205-209

29 Cheng L, Huang H, Chen S, et al. Characterization of silkworm larvae growth and properties of silk fibres after direct feeding of copper or silver nanoparticles. Mater Des, 2017, 129: 125-134

30 Wang JT, Li LL, Zhang MY, et al. Directly obtaining high strength silk fiber from silkworm by feeding carbon nanotubes. Mater Sci Eng-C, 2014, 34: 417-421

31 Wang Q, Wang C, Zhang M, et al. Feeding single-walled carbon nanotubes or graphene to silkworms for reinforced silk fibers. Nano Lett, 2016, 16: 6695-6700

$32 \mathrm{Hu}$ K, Kulkarni DD, Choi I, et al. Graphene-polymer nanocomposites for structural and functional applications. Prog Polymer Sci, 2014, 39: 1934-1972

33 Tang LC, Wan YJ, Yan D, et al. The effect of graphene dispersion on the mechanical properties of graphene/epoxy composites. Carbon, 2013, 60: 16-27

34 Hwang J, Yoon T, Jin SH, et al. Enhanced mechanical properties of graphene/copper nanocomposites using a molecular-level mixing process. Adv Mater, 2013, 25: 6724-6729

35 Lepore E, Bosia F, Bonaccorso F, et al. Spider silk reinforced by graphene or carbon nanotubes. 2D Mater, 2017, 4: 031013

36 Bacon M, Bradley SJ, Nann T. Graphene quantum dots. Part Part Syst Charact, 2014, 31: 415-428

37 Liu WW, Feng YQ, Yan XB, et al. Superior micro-supercapacitors based on graphene quantum dots. Adv Funct Mater, 2013, 23: 4111-4122

38 Shen J, Zhu Y, Yang X, et al. Graphene quantum dots: emergent nanolights for bioimaging, sensors, catalysis and photovoltaic devices. Chem Commun, 2012, 48: 3686-3699

39 Tetsuka H, Nagoya A, Fukusumi T, et al. Molecularly designed, nitrogen-functionalized graphene quantum dots for optoelectronic devices. Adv Mater, 2016, 28: 4632-4638

40 Edwards HGM, Farwell DW. Raman spectroscopic studies of silk. J Raman Spectrosc, 1995, 26: 901-909

41 Sirichaisit J, Brookes VL, Young RJ, et al. Analysis of structure/ property relationships in silkworm (Bombyx mori) and spider dragline (Nephila edulis) silks using Raman spectroscopy. Biomacromolecules, 2003, 4: 387-394

42 Keten $\mathrm{S}, \mathrm{Xu} \mathrm{Z}$, Ihle B, et al. Nanoconfinement controls stiffness, strength and mechanical toughness of $\beta$-sheet crystals in silk. Nat Mater, 2010, 9: 359-367

43 Du N, Liu XY, Narayanan J, et al. Design of superior spider silk: from nanostructure to mechanical properties. BioPhys J, 2006, 91: 4528-4535

44 Grubb DT, Ji G. Molecular chain orientation in supercontracted and re-extended spider silk. Int J Biol Macromolecules, 1999, 24: 203-210

45 Numata K, Sato R, Yazawa K, et al. Crystal structure and physical properties of Antheraea yamamai silk fibers: Long poly(alanine) sequences are partially in the crystalline region. Polymer, 2015, 77: 87-94 
Sampath S, Isdebski T, Jenkins JE, et al. X-ray diffraction study of nanocrystalline and amorphous structure within major and minor ampullate dragline spider silks. Soft Matter, 2012, 8: 6713-6722

47 Yen FS, Chen WC, Yang JM, et al. Crystallite size variations of nanosized $\mathrm{Fe}_{2} \mathrm{O}_{3}$ powders during $\gamma$ - to $\alpha$-phase transformation. Nano Lett, 2002, 2: 245-252

48 Zhang H, Bhunia K, Munoz N, et al. Linking morphology changes to barrier properties of polymeric packaging for microwave-assisted thermal sterilized food. J Appl Polym Sci, 2017, 134: 45481 Ling S, Qi Z, Knight DP, et al. Synchrotron FTIR microspectroscopy of single natural silk fibers. Biomacromolecules, 2011, 12: 3344-3349

50 Lin N, Cao L, Huang Q, et al. Functionalization of silk fibroin materials at mesoscale. Adv Funct Mater, 2016, 26: 8885-8902

$51 \mathrm{Hu}$ X, Kaplan D, Cebe P. Determining b-sheet crystallinity in fibrous proteins by thermal analysis and infrared spectroscopy. Macromolecules, 2006, 39: 6161-6170

52 Huang S, Qiu H, Lu S, et al. Study on the molecular interaction of graphene quantum dots with human serum albumin: Combined spectroscopic and electrochemical approaches. J Hazard Mater, 2015, 285: 18-26

53 Li L, Wu G, Yang G, et al. Focusing on luminescent graphene quantum dots: current status and future perspectives. Nanoscale, 2013, 5: 4015-4039

54 Ling S, Li C, Adamcik J, et al. Directed growth of silk nanofibrils on graphene and their hybrid nanocomposites. ACS Macro Lett, 2014, 3: 146-152

$55 \mathrm{Hu} \mathrm{K}$, Gupta MK, Kulkarni DD, et al. Ultra-robust graphene oxide-silk fibroin nanocomposite membranes. Adv Mater, 2013, 25: 2301-2307

56 Nova A, Keten S, Pugno NM, et al. Molecular and nanostructural mechanisms of deformation, strength and toughness of spider silk fibrils. Nano Lett, 2010, 10: 2626-2634

57 Lefèvre T, Rousseau ME, Pézolet M. Protein secondary structure and orientation in silk as revealed by Raman spectromicroscopy. BioPhys J, 2007, 92: 2885-2895

58 Hernandez Cruz D, Rousseau ME, West MM, et al. Quantitative mapping of the orientation of fibroin $\beta$-Sheets in B. mori cocoon fibers by scanning transmission X-ray microscopy. Biomacromolecules, 2006, 7: 836-843

59 Oroudjev E, Soares J, Arcidiacono S, et al. Segmented nanofibers of spider dragline silk: Atomic force microscopy and single-molecule force spectroscopy. Proc Natl Acad Sci USA, 2002, 99: 6460-6465

Acknowledgements This work was supported by the Young Elite Scientist Sponsorship Program by CAST (2015QNRC001) and the Earmarked Fund for Modern Agro-industry Technology Research System.

Author contributions Ma L designed and engineered the whole experiment. Akurugu MA and Andoh V kept, dissected and injected silkworms, measured the weight and length of silkworms as well as calculated the mortality rate of silkworms. Song J reeled silks; Ma L analyzed all the silkworm and silk samples and processed the corresponding data. Ma L wrote the paper with the support from Liu $\mathrm{H}$. Wu $\mathrm{G}$ and Li L provided experimental facilities and finance support; Wu G and $\mathrm{Li} \mathrm{L}$ gave instructions on how to keep silkworms and reel silks. All authors contributed to the general discussion.

Conflict of interest These authors declare no conflicts of interest.

Supplementary information Experimental details and supporting data are available in the online version of the paper. 

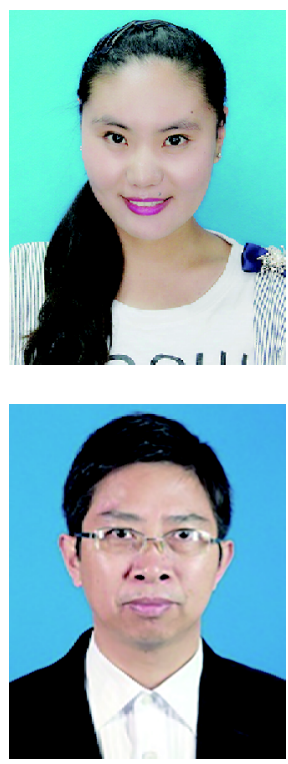

Lin Ma is an assistant research scientist at the College of Biotechnology, Jiangsu University of Science and Technology. She received her PhD (2014) from Beijing Normal University and worked in Jiangsu University of Science and Technology from then on. In 2015, she got the "Young Elite Scientist Sponsorship Program" by CAST (China Association for Science and Technology). Her current research interest focuses on the improvement of the properties of silkworm silks by using nanoparticles and the discrimination of proteins via nanoparticle-based sensor array.

Guohua Wu is a Jiangsu specially appointed professor at the College of Biotechnology in Jiangsu University of Science and Technology, and the vice director of Laboratory of Risk Assessment for Sericultural Products and Edible Insects, Ministry of Agriculture of China. His research focuses on biomaterials related with silk, especially to understand the mesoscopic structure-function relationship, and the biological effects of nanomaterials using silkworm as a model organism.

\section{摄入石墨烯量子点的家虫可直接吐出力学性能增强的虫丝}

马琳 ${ }^{1,2,3}$, Maxwell Akologo Akurugu ${ }^{1 \dagger}$, Vivian Andoh $^{1 \dagger}$, 刘海燕, 宋江超 ${ }^{1,2,3}$, 武国华 ${ }^{1,2,3^{*}}$, 李龙 ${ }^{1,2,3^{*}}$

摘要 家虫虫丝具有细淢、光鲜以及优良的力学特性, 因而在诸多领域中都有广泛应用. 许多科学家对提高家虫虫丝力学性能的研究非 常感兴趣. 本工作中, 我们通过血管注射的方式给家虫幼虫注入了石墨烯量子点(GQDs), 并且得到了力学性能增强的虫丝. GQDs可通过 淋巴循环进入到家虫的丝腺器官并影响家虫的纺丝过程. 研究发现, 当家虫的GQDs摄入量为 $0.6 \mu \mathrm{g} /$ 头时, 家虫虫丝的断裂强度、断裂伸 长率以及韧性模量可分别增强到原来的 $2.74,1.33$ 和 3.62 倍. 广角X射线散射(WAXS)结果表明GQDs-䖯丝中的 $\beta$-折叠纳米晶体的尺寸比起 空白虫丝有所降低, 红外光谱结果表明GQDs限制了丝素蛋白中无规则卷曲或者 $\alpha$-螺旋结构向 $\beta$-折叠结构的构象转变. $\beta$-折叠结构的尺寸 及含量的变化可能是引起蚕丝力学性能增强的原因所在. 我们还对GQDs的毒性以及单头家虫摄入GQDs的安全限进行了评估, 结果发现 家虫摄入 GQDs的上限为 $30.0 \mu \mathrm{g} /$ 头. 使家虫直接摄入 GQDs获得力学性能增强的虫丝的方法为生产高强度虫丝提供了一种有潜力的途径. 